\title{
On the solutions of certain fractional kinetic equations involving $k$-Mittag-Leffler function
}

\author{
P. Agarwall ${ }^{1 *}$, M. Chand ${ }^{2}$, D. Baleanu ${ }^{3,4}$, D. O'Regan ${ }^{5}$ and Shilpi Jain ${ }^{6}$
}

"Correspondence:

goyal.praveen2011@gmail.com

'Department of Mathematics, Anand International College of Engineering, Jaipur, India Full list of author information is available at the end of the article

\section{Springer}

\begin{abstract}
The aim of the present paper is to develop a new generalized form of the fractional kinetic equation involving a generalized $k$-Mittag-Leffler function $E_{k, \zeta, \eta}^{\gamma, \rho}(\cdot)$. The solutions of fractional kinetic equations are discussed in terms of the Mittag-Leffler function. Further, numerical values of the results and their graphical interpretation is interpreted to study the behavior of these solutions. The results established here are quite general in nature and capable of yielding both known and new results.
\end{abstract}

MSC: $26 \mathrm{~A} 33 ; 33 \mathrm{C} 45 ; 33 \mathrm{C} 60 ; 33 \mathrm{C} 70$

Keywords: Fractional kinetic equation; Laplace transforms; Generalized k-Mittag-Leffler function

\section{Introduction and preliminaries}

The fractional-order calculus (FOC) constitutes a branch of mathematics dealing with differentiation and integration under an arbitrary order of the operation, that is, the order can be any real or even complex number, not only the integer one [1-9]. Although the FOC represents more than 300-year-old issue [10,11], its great consequences in contemporary theoretical research and real-world applications have been widely discussed relatively recently. The idea of noninteger derivative was mentioned for the first time probably in a letter from Leibniz to L'Hospital in 1695. Later on, the pioneering works related to FOC were elaborated by personalities such as Euler, Fourier, Abel, Liouville, or Riemann. The interested reader can find a more detailed historical background of the FOC, for example, in [7].

According to [10, 12], the reason why FOC remained practically unexplored for engineering applications and why only pure mathematics was privileged to deal with it for so long time can be seen in multiple definitions of FOC, missing simple geometrical interpretation, the absence of solution methods for fractional-order differential equations and seeming adequateness of the integer-order calculus (IOC) for majority of problems. However, nowadays the situation is going better, and the FOC provides an efficient tool for many issues related to fractal dimension, "infinite memory", chaotic behavior, and so on. Thus, the FOC has already came in useful in engineering areas such as bioengineering, viscoelasticity, electronics, robotics, control theory, and signal processing [12]. Several control applications are available, for example, in [13-15].

(c) The Author(s) 2018. This article is distributed under the terms of the Creative Commons Attribution 4.0 International License (http://creativecommons.org/licenses/by/4.0/), which permits unrestricted use, distribution, and reproduction in any medium, provided you give appropriate credit to the original author(s) and the source, provide a link to the Creative Commons license, and indicate if changes were made. 
A great number of additional results of fractional calculus were presented in the twentieth century, but at this point, we only concentrate on that given by M. Caputo and first used extensively in [16]. Given a function $f$ with an $(n-1)$ th absolutely continuous derivative, Caputo defined the fractional derivative by

$$
D_{*}^{\alpha} f(x)=\frac{1}{\Gamma(n-\alpha)} \int_{0}^{t}(t-s)^{n-\alpha-1}\left(\frac{d}{d s}\right)^{n} f(s) d s,
$$

today usually named the Caputo fractional derivative. The derivative (1.1) is strongly connected to the Riemann-Liouville fractional derivative (see [17]) and is today frequently used in applications. This is because, using the Caputo derivative, we can specify the initial conditions of fractional differential equations in classical form, that is,

$$
y^{(k)}(0)=b_{k}, \quad k=0,1, \ldots, n-1,
$$

in contrast to differential equations containing the Riemann-Liouville differential operator (see [17]). Although the operator $D_{*}^{\alpha}$ is denoted today as the Caputo operator, Y.N. Rabotnov had already introduced this differential operator into the Russian viscoelastic literature in [18], a year before Caputo's paper was published.

By the second half of the twentieth century the field of fractional calculus had grown to such extent that in 1974 the first conference concerned solely with the theory and applications of fractional calculus was held in New Haven [19]. In the same year the first book on fractional calculus by Oldham and Spanier [7] was published. A number of additional books have appeared since then, the most popular by Miller and Ross [8], Samko et al. [20], and Podlubny [9]. In 1998 the first issue of the mathematical journal Fractional Calculus \& Applied Analysis was printed. This journal is solely concerned with topics on the theory of fractional calculus and its applications. Finally, in 2004 the large conference Fractional Differentiation and its Applications was held in Bordeaux, where no less than 104 talks were given in the field of fractional calculus.

From its birth—a simple question from L'Hospital to Leibniz-to its today's wide use in numerous scientific fields fractional calculus has come a long way. Even though it is nearly as old as classical calculus itself, it flourished mainly over the last decades because of its good applicability on models describing complex real-life problems (see recent works [2125]). Even though the term fractional calculus is a misnomer, we will use it throughout this text, which will be concerned with theoretical and, more importantly, numerical aspects of problems arising in this field.

The importance of fractional differential equations in the field of applied science has gained more attention not only in mathematics but also in dynamical systems, physics, control systems, and engineering, creating mathematical models of many physical phenomena. Especially, the kinetic equations describe the continuity of motion of substance. The extension and generalization of fractional kinetic equations involving fractional operators can be found in [26-32].

In view of the effectiveness and great importance of the kinetic equation in certain astrophysical problems, the authors develop a further generalized form of the fractional kinetic equation.

The fractional differential equation between the rate of change of the reaction, the destruction rate, and the production rate was established by Haubold and Mathai [26] and 
is given as follows:

$$
\frac{d \mathcal{N}}{d \tau}=-d\left(\mathcal{N}_{\tau}\right)+s\left(\mathcal{N}_{\tau}\right)
$$

where $\mathcal{N}=\mathcal{N}(\tau)$ is the rate of reaction, $d=d(\mathcal{N})$ is the rate of destruction, $s=s(\mathcal{N})$ is the rate of production, and $\mathcal{N}_{\tau}$ is the function defined by $\mathcal{N}_{\tau}\left(\tau^{*}\right)=\mathcal{N}\left(\tau-\tau^{*}\right), \tau^{*}>0$.

A particular case of (1.3) for spatial fluctuations and inhomogeneities in $\mathcal{N}(\tau)$ where the quantities are neglected is the equation

$$
\frac{d \mathcal{N}}{d \tau}=-\epsilon_{i} \mathcal{N}_{i}(\tau)
$$

with the initial condition $\mathcal{N}_{i}(\tau=0)=\mathcal{N}_{0}$ representing the number density of the species $i$ at time $\tau=0$ and $\epsilon_{i}>0$. If we remove the index $i$ and integrate the standard kinetic equation (1.4), then we have

$$
\mathcal{N}(\tau)-\mathcal{N}_{0}=-\epsilon_{0} D_{\tau}^{-1} \mathcal{N}(\tau)
$$

where ${ }_{0} D_{\tau}^{-1}$ is the particular case of the Riemann-Liouville integral operator ${ }_{0} D_{\tau}^{-v}$ defined as

$$
{ }_{0} D_{\tau}^{-v} f(\tau)=\frac{1}{\Gamma(v)} \int_{0}^{\tau}(\tau-s)^{v-1} f(s) d s \quad(\tau>0, \Re(v)>0) .
$$

A fractional generalization of the standard kinetic equation (1.5) is given by Haubold and Mathai [26] as follows:

$$
\mathcal{N}(\tau)-\mathcal{N}_{0}=-\epsilon_{0}^{v} D_{\tau}^{-1} \mathcal{N}(\tau)
$$

They obtained the solution of (1.7) as

$$
\mathcal{N}(\tau)=\mathcal{N}_{0} \sum_{k=0}^{\infty} \frac{(-1)^{k}}{\Gamma(v k+1)}(\epsilon \tau)^{v k}
$$

Saxena and Kalla [28] introduced the following fractional kinetic equation:

$$
\mathcal{N}(\tau)-\mathcal{N}_{0} f(\tau)=-\epsilon_{0}^{v} D_{\tau}^{-v} \mathcal{N}(\tau) \quad(\Re(v)>0)
$$

where $\mathcal{N}(\tau)$ denotes the number density of a given species at time $\tau, \mathcal{N}_{0}=\mathcal{N}(0)$ is the number density of that species at time $\tau=0, \epsilon$ is a constant, and $f \in \mathcal{L}(0, \infty)$. Applying the Laplace transform to (1.9) (see [27]), we have

$$
\mathcal{L}\{\mathcal{N}(\tau) ; s\}=\mathcal{N}_{0} \frac{F(s)}{1+\epsilon^{v} \mathcal{s}^{-v}}=\mathcal{N}_{0}\left(\sum_{n=0}^{\infty}\left(-\epsilon^{v}\right)^{n} s^{-v n}\right) F(s) \quad\left(n \in \mathcal{N}_{0},\left|\frac{\epsilon}{s}\right|<1\right),
$$

where the Laplace transform [33] is given by

$$
F(s)=\mathcal{L}\{\mathcal{N}(\tau) ; s\}=\int_{0}^{\infty} e^{-s \tau} f(\tau) d \tau \quad(\Re(s)>0) .
$$


We further need some additional definitions and functions. Recently, Diaz and Pariguan [34] have introduced the $k$-Pochhammer symbol defined as follows:

$$
\begin{aligned}
& (\xi)_{n, k}=\xi(\xi+k)(\xi+2 k) \cdots(\xi+(n-1) k), \\
& (\xi)_{(n+r) \rho, k}=(\xi)_{r \rho, k}(\xi+\rho r k)_{n \rho, k},
\end{aligned}
$$

where $\xi \in \mathbb{C}, k \in \mathbb{R}$, and $n \in \mathbb{N}$.

Proposition 1 Let $\xi \in \mathbb{C}$ and $k, s \in \mathbb{R}$. Then

$$
\Gamma_{s}(\xi)=\left(\frac{s}{k}\right)^{\frac{\xi}{s}-1} \Gamma_{k}\left(\frac{k \xi}{s}\right)
$$

and, in particular,

$$
\Gamma_{k}(\xi)=k^{\frac{\xi}{k}-1} \Gamma\left(\frac{\xi}{k}\right)
$$

Proposition 2 Let $\xi \in \mathbb{C}, k, s \in \mathbb{R}$ and $\xi \in \mathbb{C}$. Then

$$
(\xi)_{n \rho, s}=\left(\frac{s}{k}\right)^{n \rho}\left(\frac{k \xi}{s}\right)_{n \rho}
$$

and, in particular,

$$
(\xi)_{n \rho, k}=(k)^{n \rho}\left(\frac{\xi}{k}\right)_{n \rho} .
$$

For more detail on the $k$-Pochhammer symbol, the $k$-special function, and the fractional Fourier transform, we refer the reader to the papers by Romero et al. [35, 36].

Let $k \in \mathbb{R}, \zeta, \eta, \xi \in \mathbb{C}, \Re(\zeta)>0, \Re(\eta)>0, \Re(\xi)>0$, and $\rho \in(0,1) \cup \mathbb{N}$. Then the generalized $k$-Mittag-Leffler function, denoted by $E_{k, \zeta, \eta}^{\xi, \rho}(z)$, is defined as

$$
E_{k, \zeta, \eta}^{\xi, \rho}(z)=\sum_{n=0}^{\infty} \frac{(\xi)_{n \rho, k} z^{n}}{\Gamma_{k}(n \zeta+\eta) n !}
$$

where $(\xi)_{n \rho, k}$ denotes the $k$-Pochhammer symbol given by equation (1.17), and $\Gamma_{k}(x)$ is the $k$-gamma function given by (1.15) (also see [37]).

The generalized Pochhammer symbol is defined as (see [38, p. 22])

$$
(\xi)_{n \rho}=\frac{\Gamma(\xi+n \rho)}{\Gamma(\xi)}=\rho^{\rho n} \prod_{r=1}^{\rho}\left(\frac{\xi+r+1}{\rho}\right)_{n} \quad \text { if } \rho \in \mathbb{N} .
$$

We consider particular cases of $E_{k, \zeta, \eta}^{\xi, \rho}(z)$ :

(i) For $\rho=1$, equation (1.18) reduces to the $k$-Mittag-Leffler function (see [39]) defined as

$$
E_{k, \zeta, \eta}^{\xi, 1}(z)=\sum_{n=0}^{\infty} \frac{(\xi)_{n, k} z^{n}}{\Gamma_{k}(n \zeta+\eta) n !}=E_{k, \zeta, \eta}^{\xi}(z)
$$


(ii) For $k=1$, equation (1.18) reduces to the Mittag-Leffler function defined in [40]:

$$
E_{1, \zeta, \eta}^{\xi, \rho}(z)=\sum_{n=0}^{\infty} \frac{(\xi)_{n \rho} z^{n}}{\Gamma(n \zeta+\eta) n !}=E_{\zeta, \eta}^{\xi, \rho}(z) .
$$

(iii) For $\rho=1$ and $k=1$, (1.18) yields the Mittag-Leffler function defined by Dorrego and Cerutti [39]:

$$
E_{1, \zeta, \eta}^{\xi, 1}(z)=\sum_{n=0}^{\infty} \frac{(\xi)_{n} z^{n}}{\Gamma(n \zeta+\eta) n !}=E_{\zeta, \eta}^{\xi}(z) .
$$

(iv) For $\rho=1, k=1$, and $\xi=1$, (1.18) yields the Mittag-Leffler function (see [41]) defined as

$$
E_{1, \zeta, \eta}^{1,1}(z)=\sum_{n=0}^{\infty} \frac{z^{n}}{\Gamma(n \zeta+\eta) n !}=E_{\zeta, \eta}(z) .
$$

(v) For $\rho=1, k=1, \xi=1$, and $\eta=1$, (1.18) yields the Mittag-Leffler function (see [42]) defined as

$$
E_{1, \zeta, 1}^{1,1}(z)=\sum_{n=0}^{\infty} \frac{z^{n}}{\Gamma(n \zeta+1) n !}=E_{\zeta}(z) .
$$

Saxena et al. [43] introduce a detailed account of the Mittag-Leffler function and its applications in their survey paper.

During the first half of the twentieth century, the Mittag-Leffler (M-L) function remained almost unknown to the majority of scientists. They unjustly ignored it in many treatises on special functions, including the most common ones (as, e.g., Abramowitz and Stegun [44]). Successful applications of the Mittag-Leffler function and its generalizations, their direct involvement in problems of physics, biology, chemistry, engineering, and other applied sciences made it in the recent decades better known among the scientists. A considerable amount of literature is devoted to the investigation of the analytical properties, generalization, and the multiindex version of this function (for more detail, see [45-47]).

The objective of this paper is to derive the solution of the fractional kinetic equation involving the generalized $k$-Mittag-Leffler function. The results obtained in terms of the Mittag-Leffler function are rather general in nature, and we can easily construct various known and new fractional kinetic equations.

\section{Solution of generalized fractional kinetic equations}

In this section, we investigated the solutions of the generalized fractional kinetic equations by considering the generalized $k$-Mittag-Leffler function.

Remark 1 The solutions of the fractional kinetic equations in this section are obtained in terms of the generalized Mittag-Leffler function $E_{\zeta, \eta}(x)$ given by [42]

$$
E_{\zeta, \eta}(x)=\sum_{n=0}^{\infty} \frac{(x)^{n}}{\Gamma(\zeta n+\eta)} .
$$


Theorem 1 If $\delta>0, v>0, k \in \mathbb{R}, \zeta, \eta, \xi \in \mathbb{C} ; \Re(\zeta)>0, \Re(\eta)>0, \Re(\xi)>0$, and $\rho \in(0,1) \cup \mathbb{N}$, then the solution of the equation

$$
\mathcal{N}(\tau)-\mathcal{N}_{0} E_{k, \zeta, \eta}^{\xi, \rho}(\tau)=-\delta^{v}{ }_{0} D_{\tau}^{-v} \mathcal{N}(\tau)
$$

is given by

$$
\mathcal{N}(\tau)=\mathcal{N}_{0} \sum_{n=0}^{\infty} \frac{(\xi)_{n \tau, k}}{\Gamma_{k}(n \zeta+\eta)} \tau^{n} E_{v, n+1}\left(-\delta^{v} \tau^{v}\right) .
$$

Proof The Laplace transform of the Riemann-Liouville fractional integral operator is given by $[33,48]$

$$
\mathcal{L}\left\{{ }_{0} D_{\tau}^{-v} f(\tau) ; s\right\}=s^{-v} F(s),
$$

where $F(s)$ is defined in (1.11). Now, applying the Laplace transform to both sides of (2.2) gives

$$
\begin{gathered}
\mathcal{L}\{\mathcal{N}(\tau) ; s\}=\mathcal{N}_{0} \mathcal{L}\left\{E_{k, \zeta, \eta}^{\xi, \rho}(\tau) ; s\right\}-\delta^{v} \mathcal{L}\left\{{ }_{0} D_{\tau}^{-v} \mathcal{N}(\tau) ; s\right\} \\
\mathcal{N}(s)=\mathcal{N}_{0}\left(\int_{0}^{\infty} e^{-s \tau} \sum_{n=0}^{\infty} \frac{(\xi)_{n \tau, k}}{\Gamma_{k}(n \zeta+\eta)} \frac{\tau^{n}}{n !} d \tau\right)-\delta^{v} s^{-v} \mathcal{N}(s), \\
\mathcal{N}(s)+\delta^{v} s^{-v} \mathcal{N}(s)=\mathcal{N}_{0} \sum_{n=0}^{\infty} \frac{(\xi)_{n \tau, k}}{\Gamma_{k}(n \zeta+\eta)} \frac{1}{n !} \int_{0}^{\infty} e^{-s \tau} \tau^{n} d \tau \\
=\mathcal{N}_{0} \sum_{n=0}^{\infty} \frac{(\xi)_{n \tau, k}}{\Gamma_{k}(n \zeta+\eta)} \frac{1}{n !} \frac{\Gamma(n+1)}{s^{n+1}}, \\
\mathcal{N}(s)=\mathcal{N}_{0} \sum_{n=0}^{\infty} \frac{(\xi)_{n \tau, k}}{\Gamma_{k}(n \zeta+\eta)}\left\{s^{-(n+1)} \sum_{r=0}^{\infty}\left[-\left(\frac{s}{\delta}\right)^{-v}\right]^{r}\right\} .
\end{gathered}
$$

Taking the Laplace inverse of (2.9) and using

$$
\mathcal{L}^{-1}\left\{s^{-v} ; \tau\right\}=\frac{\tau^{v-1}}{\Gamma(v)} \quad(\Re(v)>0),
$$

we have

$$
\begin{gathered}
\mathcal{L}^{-1}\{\mathcal{N}(s)\}=\mathcal{N}_{0} \sum_{n=0}^{\infty} \frac{(\xi)_{n \tau, k}}{\Gamma_{k}(n \zeta+\eta)} \mathcal{L}^{-1}\left\{\sum_{r=0}^{\infty}(-1)^{r} \delta^{v r} s^{-(n+1+v r)}\right\} \\
\mathcal{N}(\tau)=\mathcal{N}_{0} \sum_{n=0}^{\infty} \frac{(\xi)_{n \tau, k}}{\Gamma_{k}(n \zeta+\eta)}\left\{\sum_{r=0}^{\infty}(-1)^{r} \delta^{v r} \frac{\tau^{(n+v r)}}{\Gamma(n+v r+1)}\right\} \\
=\mathcal{N}_{0} \sum_{n=0}^{\infty} \frac{(\xi)_{n \tau, k}}{\Gamma_{k}(n \zeta+\eta)} \tau^{n}\left\{\sum_{r=0}^{\infty}(-1)^{r} \frac{\delta^{v r} \tau^{v r}}{\Gamma(v r+n+1)}\right\} \\
\mathcal{N}(\tau)=\mathcal{N}_{0} \sum_{n=0}^{\infty} \frac{(\xi)_{n \tau, k}}{\Gamma_{k}(n \zeta+\eta)} \tau^{n} E_{v, n+1}\left(-\delta^{v} \tau^{v}\right) .
\end{gathered}
$$


Theorem 2 If $\delta>0, v>0, k \in \mathbb{R}, \zeta, \eta, \xi \in \mathbb{C} ; \Re(\zeta)>0, \Re(\eta)>0, \Re(\xi)>0$, and $\rho \in(0,1) \cup \mathbb{N}$, then the solution of the equation

$$
\mathcal{N}(\tau)=\mathcal{N}_{0} E_{k, \zeta, \eta}^{\xi, \rho}\left(\delta^{v} \tau^{v}\right)-\delta^{v}{ }_{0} D_{\tau}^{-v} \mathcal{N}(\tau)
$$

is given by

$$
\mathcal{N}(\tau)=\mathcal{N}_{0} \sum_{n=0}^{\infty} \frac{(\xi)_{n \tau, k}}{\Gamma_{k}(n \zeta+\eta)}\left(\delta^{v} \tau^{v}\right)^{n} E_{v, v n+1}\left(-\delta^{v} \tau^{v}\right)
$$

Theorem 3 If $\sigma>0, \delta>0, v>0, k \in \mathbb{R}, \zeta, \eta, \xi \in \mathbb{C} ; \Re(\zeta)>0, \Re(\eta)>0, \Re(\xi)>0$, and $\rho \in$ $(0,1) \cup \mathbb{N}$, then the solution of the equation

$$
\mathcal{N}(\tau)=\mathcal{N}_{0} E_{k, \zeta, \eta}^{\xi, \rho}\left(\delta^{v} \tau^{v}\right)-\sigma^{v}{ }_{0} D_{\tau}^{-v} \mathcal{N}(\tau)
$$

is given by

$$
\mathcal{N}(\tau)=\mathcal{N}_{0} \sum_{n=0}^{\infty} \frac{(\xi)_{n \tau, k}}{\Gamma_{k}(n \zeta+\eta)}\left(\delta^{v} \tau^{v}\right)^{n} E_{v, v n+1}\left(-\sigma^{v} \tau^{v}\right)
$$

Proof The proof of (2.16) and (2.18) is similar to that of (2.3).

\subsection{Numerical results}

In this section, we establish a database for numerical solutions of the kinetic equations (2.3), (2.16), and (2.18) for particular values of the parameters. Here we denote the solutions of equations (2.3) as $\mathcal{N}(\tau)=\mathcal{N}\left(\mathcal{N}_{0}, \xi, \rho, k, \zeta, \eta, \delta, v, \tau\right)$ with $\mathcal{N}_{0}=0.05, \xi=2, \rho=$ $1, k=2, \zeta=6, \eta=7, \delta=3, v=1$; for equation $(2.16)$ as $\mathcal{N}(\tau)=\mathcal{N}\left(\mathcal{N}_{0}, \xi, \rho, k, \zeta, \eta, \delta, v, \tau\right)$ with $\mathcal{N}_{0}=0.05, \xi=2, \rho=1, k=2, \zeta=6, \eta=7, \delta=3, v=5$; and for equation (2.18) as $\mathcal{N}(\tau)=\mathcal{N}\left(\mathcal{N}_{0}, \xi, \rho, k, \zeta, \eta, \delta, \sigma, v, \tau\right)$ with $\mathcal{N}_{0}=0.05, \xi=2, \rho=1, k=2, \zeta=6, \eta=7, \delta=$ $3, \sigma=3, v=7$. We obtained the database (see Table 1 ) by using these values.

In our investigation, we find that $\mathcal{N}(\tau)>0$ and the behavior of the solutions for different parameters and time intervals can be studied and observed very easily. From the database we come to the conclusion that numerical solutions of equations (2.3), (2.16), and (2.18) are approximately the same.

Table 1 Numerical values for equations (2.3), (2.16), and (2.18)

\begin{tabular}{lllll}
\hline$\tau$ & $v$ & Solution of Eq. (2.3) & Solution of Eq. (2.16) & Solution of Eq. (2.18) \\
\hline 0 & 0 & NaN & NaN & NaN \\
0.2 & 0.2 & 0.0239 & 0.0239 & 0.0248 \\
0.4 & 0.4 & 0.0132 & 0.0132 & 0.0143 \\
0.6 & 0.6 & 0.0093 & 0.0093 & 0.0106 \\
0.8 & 0.8 & 0.0071 & 0.0071 & 0.0086 \\
1 & 1 & 0.0055 & 0.0056 & 0.0073 \\
1.2 & 1.2 & 0.0045 & 0.0045 & 0.0062 \\
1.4 & 1.4 & 0.0039 & 0.0039 & 0.0052 \\
1.6 & 1.6 & 0.0040 & 0.0040 & 0.0044 \\
1.8 & 1.8 & 0.0051 & 0.0052 & 0.0034 \\
2 & 2 & 0.0078 & 0.0079 & 0.0025 \\
\hline
\end{tabular}




\section{Special cases}

If we choose $\rho=1$, then (2.3), (2.16), and (2.18) reduce to the following:

Corollary 1 If $\delta>0, v>0, k \in \mathbb{R}, \zeta, \eta, \xi \in \mathbb{C}, \Re(\zeta)>0, \Re(\eta)>0$, and $\Re(\xi)>0$, then the solution of the equation

$$
\mathcal{N}(\tau)-\mathcal{N}_{0} E_{k, \zeta, \eta}^{\xi}(\tau)=-\delta^{v}{ }_{0} D_{\tau}^{-v} \mathcal{N}(\tau)
$$

is given by

$$
\mathcal{N}(\tau)=\mathcal{N}_{0} \sum_{n=0}^{\infty} \frac{(\xi)_{n, k}}{\Gamma_{k}(n \zeta+\eta)} \tau^{n} E_{v, n+1}\left(-\delta^{v} \tau^{v}\right) .
$$

Corollary 2 If $\delta>0, v>0, k \in \mathbb{R}, \zeta, \eta, \xi \in \mathbb{C}, \Re(\zeta)>0, \Re(\eta)>0$, and $\Re(\xi)>0$, then the solution of the equation

$$
\mathcal{N}(\tau)=\mathcal{N}_{0} E_{k, \zeta, \eta}^{\xi}\left(\delta^{v} \tau^{v}\right)-\delta^{v}{ }_{0} D_{\tau}^{-v} \mathcal{N}(\tau)
$$

is given by

$$
\mathcal{N}(\tau)=\mathcal{N}_{0} \sum_{n=0}^{\infty} \frac{(\xi)_{n, k}}{\Gamma_{k}(n \zeta+\eta)}\left(\delta^{v} \tau^{v}\right)^{n} E_{v, v n+1}\left(-\delta^{v} \tau^{v}\right) .
$$

Corollary 3 If $\sigma>0, \delta>0, v>0, k \in \mathbb{R}, \zeta, \eta, \xi \in \mathbb{C}, \Re(\zeta)>0, \Re(\eta)>0$, and $\Re(\xi)>0$, then the solution of the equation

$$
\mathcal{N}(\tau)=\mathcal{N}_{0} E_{k, \zeta, \eta}^{\xi}\left(\delta^{v} \tau^{v}\right)-\sigma^{v}{ }_{0} D_{\tau}^{-v} \mathcal{N}(\tau)
$$

is given by

$$
\mathcal{N}(\tau)=\mathcal{N}_{0} \sum_{n=0}^{\infty} \frac{(\xi)_{n, k}}{\Gamma_{k}(n \zeta+\eta)}\left(\delta^{v} \tau^{v}\right)^{n} E_{v, v n+1}\left(-\sigma^{v} \tau^{v}\right)
$$

If we choose $k=1$, then (2.3), (2.16), and (2.18) reduce to the following:

Corollary 4 If $\delta>0, v>0, \zeta, \eta, \xi \in \mathbb{C}, \Re(\zeta)>0, \Re(\eta)>0, \Re(\xi)>0$, and $\rho \in(0,1) \cup \mathbb{N}$, then the solution of the equation

$$
\mathcal{N}(\tau)-\mathcal{N}_{0} E_{\zeta, \eta}^{\xi, \rho}(\tau)=-\delta^{v}{ }_{0} D_{\tau}^{-v} \mathcal{N}(\tau)
$$

is given by

$$
\mathcal{N}(\tau)=\mathcal{N}_{0} \sum_{n=0}^{\infty} \frac{(\xi)_{n q, k}}{\Gamma_{k}(n \zeta+\eta)} \tau^{n} E_{v, n+1}\left(-\delta^{v} \tau^{v}\right) .
$$


Corollary 5 If $\delta>0, v>0, \zeta, \eta, \xi \in \mathbb{C}, \Re(\zeta)>0, \Re(\eta)>0, \Re(\xi)>0$, and $\rho \in(0,1) \cup \mathbb{N}$, then the solution of the equation

$$
\mathcal{N}(\tau)-\mathcal{N}_{0} E_{\zeta, \eta}^{\xi, \rho}\left(\delta^{v} \tau^{v}\right)=-\delta^{v}{ }_{0} D_{\tau}^{-v} \mathcal{N}(\tau)
$$

is given by

$$
\mathcal{N}(\tau)=\mathcal{N}_{0} \sum_{n=0}^{\infty} \frac{(\xi)_{n q, k}}{\Gamma_{k}(n \zeta+\eta)}\left(\delta^{v} \tau^{v}\right)^{n} E_{v, n+1}\left(-\delta^{v} \tau^{v}\right)
$$

Corollary 6 If $\sigma>0, \delta>0, v>0, \zeta, \eta, \xi \in \mathbb{C}, \Re(\zeta)>0, \Re(\eta)>0, \Re(\xi)>0$, and $\rho \in(0,1) \cup \mathbb{N}$, then the solution of the equation

$$
\mathcal{N}(\tau)-\mathcal{N}_{0} E_{\zeta, \eta}^{\xi, \rho}\left(\delta^{v} \tau^{v}\right)=-\sigma^{v}{ }_{0} D_{\tau}^{-v} \mathcal{N}(\tau)
$$

is given by

$$
\mathcal{N}(\tau)=\mathcal{N}_{0} \sum_{n=0}^{\infty} \frac{(\xi)_{n q, k}}{\Gamma_{k}(n \zeta+\eta)}\left(\delta^{v} \tau^{v}\right)^{n} E_{v, n+1}\left(-\sigma^{v} \tau^{v}\right)
$$

If we choose $\rho=1$ and $k=1$, then (2.3), (2.16), and (2.18) reduce to the following:

Corollary 7 If $\delta>0, v>0, \zeta, \eta, \xi \in \mathbb{C}, \Re(\zeta)>0, \Re(\eta)>0$, and $\Re(\xi)>0$, then the solution of the equation

$$
\mathcal{N}(\tau)-\mathcal{N}_{0} E_{\zeta, \eta}^{\xi}(\tau)=-\delta^{v}{ }_{0} D_{\tau}^{-v} \mathcal{N}(\tau)
$$

is given by

$$
\mathcal{N}(\tau)=\mathcal{N}_{0} \sum_{n=0}^{\infty} \frac{(\xi)_{n}}{\Gamma(n \zeta+\eta)} \tau^{n} E_{v, n+1}\left(-\delta^{v} \tau^{v}\right) .
$$

Corollary 8 If $\delta>0, v>0, \zeta, \eta, \xi \in \mathbb{C}, \Re(\zeta)>0, \Re(\eta)>0$, and $\Re(\xi)>0$, then the solution of the equation

$$
\mathcal{N}(\tau)=\mathcal{N}_{0} E_{\zeta, \eta}^{\xi}\left(\delta^{v} \tau^{v}\right)-\delta^{v}{ }_{0} D_{\tau}^{-v} \mathcal{N}(\tau)
$$

is given by

$$
\mathcal{N}(\tau)=\mathcal{N}_{0} \sum_{n=0}^{\infty} \frac{(\xi)_{n}}{\Gamma(n \zeta+\eta)}\left(\delta^{v} \tau^{v}\right)^{n} E_{v, v n+1}\left(-\delta^{v} \tau^{v}\right)
$$

Corollary 9 If $\sigma>0, \delta>0, v>0, \zeta, \eta, \xi \in \mathbb{C}, \mathfrak{R}(\zeta)>0, \Re(\eta)>0$, and $\Re(\xi)>0$, then the solution of the equation

$$
\mathcal{N}(\tau)=\mathcal{N}_{0} E_{\zeta, \eta}^{\xi}\left(\delta^{v} \tau^{v}\right)-\sigma^{v}{ }_{0} D_{\tau}^{-v} \mathcal{N}(\tau)
$$


is given by

$$
\mathcal{N}(\tau)=\mathcal{N}_{0} \sum_{n=0}^{\infty} \frac{(\xi)_{n}}{\Gamma(n \zeta+\eta)}\left(\delta^{v} \tau^{v}\right)^{n} E_{v, v n+1}\left(-\sigma^{v} \tau^{v}\right)
$$

If we choose $\rho=1, k=1$, and $\xi=1$, then (2.3), (2.16), and (2.18) reduce to the following: Corollary 10 If $\delta>0, v>0, \zeta, \eta \in \mathbb{C}, \Re(\zeta)>0, \Re(\eta)>0$, then the solution of the equation

$$
\mathcal{N}(\tau)-\mathcal{N}_{0} E_{\zeta, \eta}(\tau)=-\delta^{v}{ }_{0} D_{\tau}^{-v} \mathcal{N}(\tau)
$$

is given by

$$
\mathcal{N}(\tau)=\mathcal{N}_{0} \sum_{n=0}^{\infty} \frac{\tau^{n}}{\Gamma(n \zeta+\eta)} E_{v, n+1}\left(-\delta^{v} \tau^{v}\right)
$$

Corollary 11 If $\delta>0, v>0, \zeta, \eta \in \mathbb{C}, \Re(\zeta)>0, \Re(\eta)>0$, then the solution of the equation

$$
\mathcal{N}(\tau)=\mathcal{N}_{0} E_{\zeta, \eta}\left(\delta^{v} \tau^{v}\right)-\delta^{v}{ }_{0} D_{\tau}^{-v} \mathcal{N}(\tau)
$$

is given by

$$
\mathcal{N}(\tau)=\mathcal{N}_{0} \sum_{n=0}^{\infty} \frac{\left(\delta^{v} \tau^{v}\right)^{n}}{\Gamma(n \zeta+\eta)} E_{v, v n+1}\left(-\delta^{v} \tau^{v}\right)
$$

Corollary 12 If $\sigma>0, \delta>0, v>0, \zeta, \eta \in \mathbb{C}, \Re(\zeta)>0, \Re(\eta)>0$, then the solution of the equation

$$
\mathcal{N}(\tau)=\mathcal{N}_{0} E_{\zeta, \eta}\left(\delta^{v} \tau^{v}\right)-\sigma_{0}^{v} D_{\tau}^{-v} \mathcal{N}(\tau)
$$

is given by

$$
\mathcal{N}(\tau)=\mathcal{N}_{0} \sum_{n=0}^{\infty} \frac{\left(\delta^{v} \tau^{v}\right)^{n}}{\Gamma(n \zeta+\eta)} E_{v, v n+1}\left(-\sigma^{v} \tau^{v}\right)
$$

If we choose $\rho=1, k=1, \xi=1$, and $\eta=1$, then (2.3), (2.16), and (2.18) reduce to the following:

Corollary 13 If $\delta>0, v>0, \zeta \in \mathbb{C}, \Re(\zeta)>0$, then the solution of the equation

$$
\mathcal{N}(\tau)-\mathcal{N}_{0} E_{\zeta}(\tau)=-\delta^{v}{ }_{0} D_{\tau}^{-v} \mathcal{N}(\tau)
$$

is given by

$$
\mathcal{N}(\tau)=\mathcal{N}_{0} \sum_{n=0}^{\infty} \frac{\tau^{n}}{\Gamma(n \zeta+1)} E_{v, n+1}\left(-\delta^{v} \tau^{v}\right)
$$


Corollary 14 If $\delta>0, v>0, \zeta \in \mathbb{C}, \Re(\zeta)>0$, then the solution of the equation

$$
\mathcal{N}(\tau)=\mathcal{N}_{0} E_{\zeta}\left(\delta^{v} \tau^{v}\right)-\delta^{v}{ }_{0} D_{\tau}^{-v} \mathcal{N}(\tau)
$$

is given by

$$
\mathcal{N}(\tau)=\mathcal{N}_{0} \sum_{n=0}^{\infty} \frac{\left(\delta^{v} \tau^{v}\right)^{n}}{\Gamma(n \zeta+1)} E_{v, v n+1}\left(-\delta^{v} \tau^{v}\right)
$$

Corollary 15 If $\sigma>0, \delta>0, v>0, \zeta \in \mathbb{C} ; \Re(\zeta)>0$, then the solution of the equation

$$
\mathcal{N}(\tau)=\mathcal{N}_{0} E_{\zeta}\left(\delta^{v} \tau^{v}\right)-\sigma^{v}{ }_{0} D_{\tau}^{-v} \mathcal{N}(\tau)
$$

is given by

$$
\mathcal{N}(\tau)=\mathcal{N}_{0} \sum_{n=0}^{\infty} \frac{\left(\delta^{v} \tau^{v}\right)^{n}}{\Gamma(n \zeta+1)} E_{v, v n+1}\left(-\sigma^{v} \tau^{v}\right)
$$

If we choose $\rho=1, k=1, \xi=1, \zeta=0$, and $\eta=1$, then (2.3), (2.16), and (2.18) reduce to the following:

Corollary 16 If $\delta>0, v>0$, then the solution of the equation

$$
\mathcal{N}(\tau)-\mathcal{N}_{0} e^{\tau}=-\delta^{v}{ }_{0} D_{\tau}^{-v} \mathcal{N}(\tau)
$$

is given by

$$
\mathcal{N}(\tau)=\mathcal{N}_{0} e^{\tau} E_{v, n+1}\left(-\delta^{v} \tau^{v}\right)
$$

Corollary 17 If $\delta>0, v>0$, then the solution of the equation

$$
\mathcal{N}(\tau)=\mathcal{N}_{0} e^{\left(\delta^{v} \tau^{v}\right)}-\delta^{v}{ }_{0} D_{\tau}^{-v} \mathcal{N}(\tau)
$$

is given by

$$
\mathcal{N}(\tau)=\mathcal{N}_{0} e^{\left(\delta^{v} \tau^{v}\right)^{n}} E_{v, v n+1}\left(-\delta^{v} \tau^{v}\right)
$$

Corollary 18 If $\sigma>0, \delta>0, v>0$, then the solution of the equation

$$
\mathcal{N}(\tau)=\mathcal{N}_{0} e^{\left(\delta^{v} \tau^{v}\right)}-\sigma^{v}{ }_{0} D_{\tau}^{-v} \mathcal{N}(\tau)
$$

is given by

$$
\mathcal{N}(\tau)=\mathcal{N}_{0} e^{\left(\delta^{v} \tau^{v}\right)^{n}} E_{v, v n+1}\left(-\sigma^{v} \tau^{v}\right)
$$


Conflict of interest

The authors declare that there is no conflict of interests.

\section{Funding}

No funding.

\section{Competing interests}

The authors declare that they have no competing interests.

\section{Authors' contributions}

All authors contributed equally to the manuscript. All authors read and approved the final manuscript.

\section{Author details}

${ }^{1}$ Department of Mathematics, Anand International College of Engineering, Jaipur, India. ${ }^{2}$ Department of Applied Sciences, Guru Kashi University, Bathinda, India. ${ }^{3}$ Department of Mathematics, Faculty of Arts and Sciences, Cankaya University, Ankara, Turkey. ${ }^{4}$ Mathematics, Institute of Space Sciences, Bucharest-Magurele, Romania. ${ }^{5}$ School of Mathematics, Statistics and Applied Mathematics, National University of Ireland, Galway, Ireland. ${ }^{6}$ Department of Mathematics, Poornima College of Engineering, Jaipur, India.

\section{Publisher's Note}

Springer Nature remains neutral with regard to jurisdictional claims in published maps and institutional affiliations.

Received: 6 September 2017 Accepted: 4 July 2018 Published online: 20 July 2018

\section{References}

1. Agarwal, P.: Further results on fractional calculus of Saigo operators. Appl. Appl. Math. 7(2), 585-594 (2012)

2. Agarwal, P.: Generalized fractional integration of the $\bar{H}$-function. Le Matematiche LXVII, $107-118$ (2012)

3. Agarwal, P., Choi, J.: Fractional calculus operators and their images formulas. J. Korean Math. Soc. 53(5), 1183-1210 (2016)

4. Agarwal, P., Jain, S.: Further results on fractional calculus of Srivastava polynomials. Bull. Math. Anal. Appl. 3(2), 167-174 (2011)

5. Agarwal, P., El-Sayed, A.A.: Non-standard finite difference and Chebyshev collocation methods for solving fractional diffusion equation. Phys. A, Stat. Mech. Appl. 500, 40-49 (2018)

6. Agarwal, P., Nieto, J.J., Luo, M.J.: Extended Riemann-Liouville type fractional derivative operator with applications. Open Math. 15(1), 1667-1681 (2017)

7. Oldham, K., Spanier, J.: Fractional Calculus: Theory and Applications of Differentiation and Integration of Arbitrary Order. Academic Press, New York (1974)

8. Miller, K.S., Ross, B.: An Introduction to the Fractional Calculus and Fractional Differential Equations. Wiley, New York (1993)

9. Podlubny, I.: Fractional Differential Equations. Academic Press, New York (1999)

10. Chen, Y., Petráš, I., Xue, D.: Fractional order control: a tutorial. In: Proceedings of 2009 American Control Conference, St. Louis, MO, USA (2009)

11. Petráš, I.: Stability of fractional-order systems with rational orders: a survey. Fract. Calc. Appl. Anal. 12, 269-298 (2009)

12. Gutiérrez, R.E., Rosário, J.M., Machado, J.T.: Ractional order calculus: basic concepts and engineering applications. Math. Probl. Eng. 2010, Article ID 375858 (2010) https://doi.org/10.1155/2010/375858

13. Axtell, M., Bise, M.E.: Fractional calculus applications in control systems. In: Proceedings of the 1990 National Aerospace and Electronics Conference, Dayton, OH, USA (1990)

14. Hamamci, S.E.: Stabilization using fractional order PI and PID controllers. Nonlinear Dyn. 51, 329-343 (2008). https://doi.org/10.1007/s11071-007-9214-5

15. Hamamci, S.E., Koksal, M.: Calculation of all stabilizing fractional-order PD controllers for integrating time delay systems. Comput. Math. Appl. 59, 1621-1629 (2010). https://doi.org/10.1016/j.camwa.2009.08.049

16. Caputo, M.: Linear models of dissipation whose $Q$ is almost frequency independent-II. Geophys. J. R. Astron. Soc. 13, 529-539 (1967)

17. Kilbas, A.A., Srivastava, H.M., Trujillo, J.J.: Theory and Applications of Fractional Differential Equations. North-Holland Mathematics Studies, vol. 204. Elsevier, Amsterdam (2006)

18. Rabotnov, Y.N.: Creep Problems in Structural Members. North-Holland Series in Applied Mathematics and Mechanics, vol. 7 (1969)

19. Ross, B.: Fractional calculus and its applications. In: Proceedings of the International Conference Held at the University of New Haven, West Haven, CT, USA (1974)

20. Samko, S.G., Kilbas, A.A., Marichev, O.I.: Fractional Integrals and Derivatives: Theory and Applications. Gordon \& Breach, New York (1993)

21. Escamilla, A.C., Gómez-Aguilar, J.F., Baleanu, D., Córdova-Fraga, T., Escobar-Jiménez, R.F., Olivares-Peregrino, V.H., Qurashi, M.M.A.: Bateman-Feshbach-Tikochinsky and Caldirola-Kanai oscillators with new fractional differentiation Entropy 19(2), 1-13 (2017)

22. Escamilla, A.C., Torres, F., Gómez-Aguilar, J.F., Escobar-Jiménez, R.F., Guerrero-Ramírez, G.V.: On the trajectory tracking control for an SCARA robot manipulator in a fractional model driven by induction motors with PSO tuning. Multibody Syst. Dyn. 43, 257-277 (2017) https://doi.org/10.1007/s11044-017-9586-3

23. Escamilla, A.C., Gómez-Aguilar, J.F., Torres, L., Escobar-Jiménez, R.F.: A numerical solution for a variable-order reaction-diffusion model by using fractional derivatives with non-local and non-singular kernel. Physica A 491, 406-424 (2018). https://doi.org/10.1016/j.physa.2017.09.014 
24. Gómez-Aguilar, J.F.: Chaos in a nonlinear Bloch system with Atangana-Baleanu fractional derivatives. Numer. Methods Partial Differ. Equ. 33, 1-23 (2017). https://doi.org/10.1002/num.22219

25. Gómez-Aguilar, J.F., Yépez-Martínez, H., Torres-Jiménez, J., Córdova-Fraga, T., Escobar-Jiménez, R.F., Olivares-Peregrino, V.H.: Homotopy perturbation transform method for nonlinear differential equations involving to fractional operator with exponential kernel. Adv. Differ. Equ. 2017, 68 (2017). https://doi.org/10.1186/s13662-017-1120-7

26. Haubold, H.J., Mathai, A.M.: The fractional kinetic equation and thermonuclear functions. Astrophys. Space Sci. 327 53-63 (2010)

27. Chand, M., Agarwal, P., Hammouch, Z:: Certain sequences involving product of k-Bessel function. Int. J. Appl. Comput. Math. 4, 101 (2018)

28. Saxena, R.K., Kalla, S.L.: On the solutions of certain fractional kinetic equations. Appl. Math. Comput. 199, 504-511 (2008)

29. Nisar, K.S., Purohit, S.D., Mondal, S.R.: Generalized fractional kinetic equations involving generalized Struve function of the first kind. J. King Saud Univ., Sci. 28, 167-171 (2016)

30. Purohit, S.D.: Solutions of fractional partial differential equations of quantum mechanics. Adv. Appl. Math. Mech. 5(5), 639-651 (2013)

31. Chouhan, A., Purohit, S.D., Saraswat, S.: An alternative method for solving generalized differential equations of fractional order. Kragujev. J. Math. 37(2), 299-306 (2013)

32. Purohit, S.D., Kalla, S.L.: On fractional partial differential equations related to quantum mechanics. J. Phys. A, Math. Theor. 44, 4 (2011)

33. Spiegel, M.R.: Theory and Problems of Laplace Transforms. Schaums Outline Series. McGraw-Hill, New York (1965)

34. Diaz, R., Pariguan, E.: On hypergeometric functions and k-Pochhammer symbol. Divulg. Mat. 15(2), 179-192 (2007)

35. Romero, L., Cerutti, R.: Fractional Fourier transform and special k-function. Int. J. Contemp. Math. Sci. 7(4), 693-704 (2012)

36. Romero, L., Cerutti, R., Luque, I.: A new fractional Fourier transform and convolutions products. Int. J. Pure Appl. Math. Sci. 66, 397-408 (2011)

37. Srivastava, H.M., Tomovski, Ž.: Fractional calculus with an integral operator containing a generalized Mittag-Leffler function in the kernel. Appl. Math. Comput. 211, 198-210 (2009)

38. Rainville, E.D.: Special Functions. Macmillan Co., New York (1963)

39. Dorrego, G.A., Cerutti, R.A.: The k-Mittag-Leffler function. Int. J. Contemp. Math. Sci. 7(15), 705-716 (2012)

40. Shukla, A.K., Prajapati, J.C.: On the generalization of Mittag-Leffler function and its properties. J. Math. Anal. Appl. 336, 797-811 (2007)

41. Wiman, A.: Uber den fundamental Satz in der Theories der Funktionen $E_{\zeta}$ (z). Acta Math. 29, 191-201 (1905)

42. Mittag-Leffler, G.M.: Sur la représentation analytique d'une fonction monogène (cinquième note). Acta Math. 29 , 101-181 (1905)

43. Saxena, R.K., Ram, J., Vishnoi, M.: Certain properties of a generalized Mittag-Leffler function. J. Indian Acad. Math. 32(2), 663-675 (2010)

44. Abramovitz, M., Stegun, I.A.: Handbook of Mathematical Functions. National Bureau of Standards, Washington (1972)

45. Saxena, R.K., Kalla, S.L., Saxena, R.: Multivariate analogue of generalized Mittag-Leffler function. Integral Transforms Spec. Funct. 22(7), 533-548 (2011)

46. Kalla, S.L., Haidey, V., Virchenko, N.: A generalized multiparameter function of Mittag-Leffler type. Integral Transforms Spec. Funct. 23(12), 901-911 (2012)

47. Kilbas, A.A., Koroleva, A.A., Rogosin, S.V.: Multi-parametric Mittag-Leffler functions and their extension. Fract. Calc. Appl. Anal. 16(2), 378-404 (2013)

48. Erdelyi, A., Magnus, W., Oberhettinger, F., Tricomi, F.G.: Tables of Integral Transforms, vol. 1. McGraw-Hill, New York (1954)

\section{Submit your manuscript to a SpringerOpen ${ }^{\circ}$ journal and benefit from:}

- Convenient online submission

- Rigorous peer review

- Open access: articles freely available online

- High visibility within the field

- Retaining the copyright to your article

Submit your next manuscript at $\boldsymbol{s p r i n g e r o p e n . c o m ~}$ 